\title{
Granulomatous cervicofacial lymphadenitis in children: a nine-year study in Singapore
}

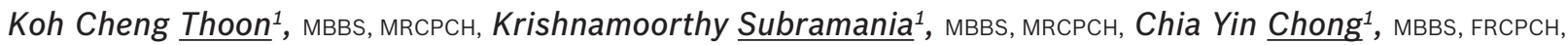
Kenneth Tou En Chang', MBChB, FRCPath, Nancy Wen Sim $\underline{T e e}^{2}$, MBBS, FRCPA

INTRODUCTION Granulomatous cervicofacial lymphadenitis (GCL) is not uncommon in children. Nontuberculous mycobacteria (NTM) seem to be the predominant cause. We sought to study the clinical and microbiological profile of patients with GCL, and identify features that may impact outcome.

METHODS Children aged < 16 years who presented to KK Women's and Children's Hospital, Singapore, between January 1998 and December 2006, and who had GCL were identified from laboratory records. Clinical and laboratory data was collected and analysed for risk factors for patients with positive lymph node cultures, and for patients with and without recurrence after treatment.

RESULTS In all, 60 children were identified, with a median age of 56 (interquartile range [IQR] 34-101) months. Median duration of symptoms before presentation was 5 (IQR 4-8) weeks. The majority presented with single (73.3\%) or unilateral $(96.7 \%)$ lymphadenopathy, located in the submandibular, preauricular/parotid or infra-/post-auricular region (76.7\%). Out of 51 patients, 26 (51.0\%) had a tuberculin skin test reading of $\geq 10 \mathrm{~mm}$. Out of 52 patients, 10 (19.2\%) had positive mycobacterial cultures, which included seven isolates of NTM. Out of 34 cases, tuberculous polymerase chain reaction was positive in $11(32.4 \%)$. With regard to recurrence after initial treatment, age $<5$ years at presentation was found to be a predictor for recurrence $(p=0.008)$, while initial complete excision of affected nodes predicted no recurrence $(p=0.003)$. CONCLUSION In our study, younger age was noted to be associated with a higher chance of recurrence, while complete excision of the involved node at initial presentation predicted non-recurrence.

Keywords: cervicofacial lymphadenitis, granulomatous, nontuberculous mycobacteria, Singapore

\section{INTRODUCTION}

Not uncommon in children, granulomatous lesions of the head and neck often present as either lymphadenopathy or subacute or chronic lymphadenitis for investigation. ${ }^{(1,2)}$ Several historical reviews have shown that granulomatous diseases may account for $7 \%-32 \%$ of biopsies of cervical masses or lymph nodes in children, and that granulomatous inflammation may be the second most common finding in histological examinations of peripheral lymph nodes. ${ }^{(2-6)}$

The granulomatous response is a generic reaction to the presence of a persistent endogenous or exogenous insoluble irritant, characterised by the accumulation of modified macrophages (i.e. epithelioid histiocytes), and is dependent on the state of the host's immune system. ${ }^{(5)}$ In adults, tuberculosis (TB), nontuberculous mycobacteria (NTM), sarcoidosis, fungal infections, rheumatoid disease and foreign body inclusions cause most cases of granulomatous lymphadenitis. Contrastingly, historical series have shown NTM to account for most granulomatous lesions of the head and neck in children. ${ }^{(7-9)}$ While one series showed a predominance of either Mycobacterium avium complex (MAC) or Mycobacterium scrofulaceum, Mycobacterium malmoense has been reported by others as the second most common pathogen after MAC in northern Europe and the United Kingdom. . $^{(10-14)}$

Distinguishing between the NTM and tuberculous forms of lymphadenitis is important because both conditions can appear identical histologically. Currently, NTM lymphadenitis in immunocompetent children is best treated with complete excision, whereas antituberculous drugs are required in tuberculous lymphadenitis. ${ }^{(9)}$ As opposed to tuberculous lymphadenitis, NTM lymphadenitis is not infectious, hence notification with contact tracing is not required. However, differentiation between these two forms of lymphadenitis is difficult in practice because: (a) NTM infections are fastidious, with cultures often taking several weeks to grow, and are tested positive in only half of the cases; $^{(5,6,11)}$ (b) histopathological examination and positive acidfast bacilli (AFB) may be nonspecific for either condition; ${ }^{(5,15)}$ and (c) other diagnostic techniques such as the use of skin testing with tuberculin (i.e. purified protein derivative [PPD]-T), NTM antigens (i.e. PPD-B, PPD-Y, PPD-G) or polymerase chain reaction (PCR) either have only moderate predictive values or are associated with significant adverse reactions, including skin ulcerations (as in the case of skin testing with NTM antigens). ${ }^{(9,10-11,16)}$

Several authors have adopted and modified Starke's recommendations and guidelines provided by the American Thoracic Society for the management of NTM lymphadenitis, but are not aware of any local series of granulomatous cervicofacial lymphadenitis (GCL) previously. ${ }^{(11,17)}$ Hence, this study aimed to describe the clinical and microbiological epidemiology of patients in our institution with GCL, and identify any features that may impact patient outcome.

${ }^{1}$ Department of Paediatric Medicine, ${ }^{2}$ Department of Pathology and Laboratory Medicine, KK Women's and Children's Hospital, Singapore

Correspondence: Dr Koh Cheng Thoon, Head and Senior Consultant, Infectious Disease Service, Department of Paediatric Medicine, Level 3, Children's Tower, KK Women's and Children's Hospital, 100 Bukit Timah Road, Singapore 229899. Thoon.Koh.Cheng@kkh.com.sg 


\section{METHODS}

This was a retrospective study performed at KK Women's and Children's Hospital, Singapore, between January 1998 and December 2006. All children aged $<16$ years with GCL were eligible for inclusion in the study and were identified from: (a) children referred to the Paediatric Infectious Disease Services for cervicofacial lymphadenopathy with biopsies showing granulomatous inflammation; and (b) histopathology records of children with cervicofacial lymph node biopsies showing granulomatous inflammation, obtained from the Department of Pathology and Laboratory Medicine. The case notes of the identified patients were retrieved and the following data was collected: (a) demographic details; (b) clinical details (i.e. patient history, examination findings and tuberculin skin test [TST] sizes); (c) chest radiography results; (d) laboratory results (i.e. haematology, biochemistry, serology, microbiology and histopathology); (e) treatment modality, whether surgical, medical, or conservative, including the type of surgery performed and type of antibiotics used, if any; and (f) treatment outcome (i.e. whether there was recurrence or not). Local recurrence was defined as either clinical redevelopment of lymphadenopathy or the presence of a discharging fistula in the same cervicofacial region within the follow-up period (as assessed clinically or ultrasonographically). Complete excision biopsy was the complete removal of the affected lymph nodes, with no visible affected lymph node tissue left in situthis was determined by the surgeon at the time of the procedure. Ethics approval was obtained from the Institutional Review Board.

TSTs were performed by trained medical and nursing staff using the Mantoux method $-0.1 \mathrm{~mL}$ (or 2 tuberculin units) of Tuberculin PPD RT 23 SSI (Statens Serum Institut, Copenhagen, Denmark) was injected intradermally, resulting in a transient raised papule of $8-10 \mathrm{~mm}$ in diameter. Patients were required to return within 48-72 hours, and TST size was evaluated by measuring the transverse diameter of the indurated portion only.

Descriptive statistics were applied to categorical and continuous variables. Chi-square and Fisher's exact tests were applied to categorical variables, and Mann-Whitney $U$ test was applied to continuous variables. With specific microbiology (focusing on either tuberculosis or NTM) and recurrence of disease as outcomes of interest, and clinical characteristics as independent variables, binary logistic regression analysis was performed on variables found to be significant on univariate analysis. A p-value $<0.05$ was considered significant. Data analysis was performed using the Statistical Package for the Social Sciences for Windows version 12 (SPSS Inc, Chicago, IL, USA).

\section{RESULTS}

We identified 60 eligible patients during the study period who were included in the present study. While there was an average of three confirmed cases per year during the first five-year period from 1998 to 2002, 2003-2006 showed an average of about 12 cases per year, representing a four-fold increase. Demographically, of these 60 patients, there were more children in the age group $2-5$ years $(\mathrm{n}=32 ; 53.3 \%)$, with only $7(11.7 \%)$ aged $>10$ years (Fig. 1). Overall, $41(68.3 \%)$ were male, and

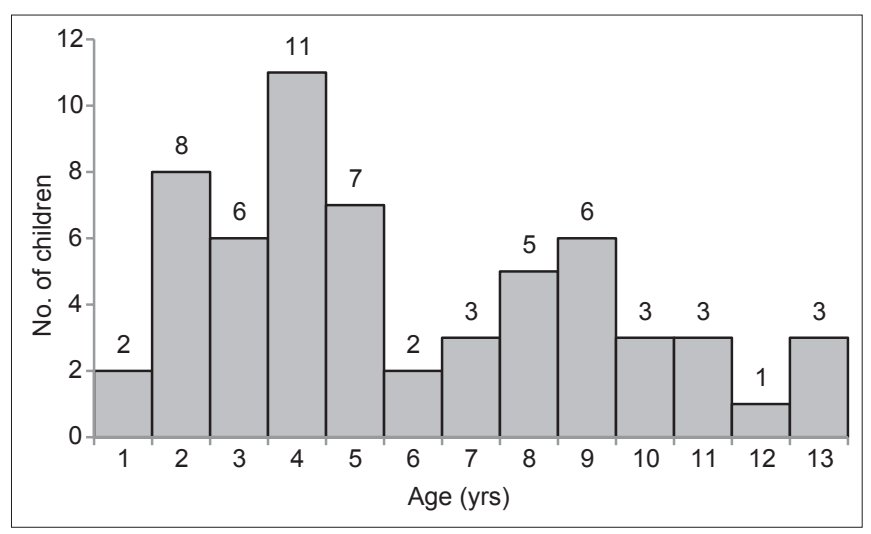

Fig. 1 Graph shows the age distribution of children who presented with granulomatous cervicofacial lymphadenitis.

$46(76.7 \%), 8(13.3 \%), 3(5.0 \%)$ and $3(5.0 \%)$ children were of Chinese, Malay, Indian and Eurasian/mixed racial ethnicity, respectively.

The median duration of symptoms before clinical presentation and diagnostic workup was 5 (interquartile range [IQR] 4-8) weeks. Of all the cases, $73.3 \%$ presented with an isolated palpable lymph node enlargement, and the rest presented with multiple lymphadenopathies. The average lymph node size was $1.5-3 \mathrm{~cm}$ in $38(63.3 \%)$ of cases; $30 \%$ of cases had nodes measuring $>3 \mathrm{~cm}$ in diameter. Local discolouration was noted only in 29 (48.3\%) of cases, with 23 of these 29 cases being erythematous, while the remaining had purple/blue skin discolouration. Of the 58 cases for which tenderness was noted at initial presentation, 39 (67.2\%) were non-tender. There was no local warmth noted in the majority ( $n=52 ; 86.7 \%$ ) of the 60 patients, which suggests the possible absence of classic bacterial inflammation. In all of the 60 patients, submandibular and preauricular/parotid lymphadenopathy accounted for 37 (61.7\%), followed by infra-/postauricular and cervical lymphadenopathies both accounting for 9 (15.0\%) cases each, with no deep extension noted in any of the cases. Out of the 60 cases, lymph node consistency was varied, with evidence of fluctuance in 13 (21.7\%); 18 (30.0\%) were soft, while 29 (48.3\%) were noted to be firm to hard. Out of the 59 patients in whom data regarding the presence of pyrexia was available, fever lasting $1-7$ days was present in $9(15.3 \%)$, and only $6(10.2 \%)$ of these 59 had respiratory symptoms (i.e. cough) on presentation. The median duration of systemic symptoms was 3 (IQR 2-7) days. None of our patients had a history of night sweats, lethargy or recent weight loss. Contact history for TB was present in 10 out of the 59 (16.9\%) cases where the relevant data was available; however, only two cases had a significant contact history. Out of the total 60 patients, 14 (23.3\%) of cases had a travel history of about a week's duration with no definite history of contact with ill persons during the travel period. In these 60 patients, $6(10.0 \%)$ had a recent history of toothache, dental procedures or dental caries.

The relevant serological tests were carried out in a total of 43 patients, all of whom had no abnormal serology positive for Epstein-Barr virus, cytomegalovirus, Bartonella, Toxoplasma, mumps or human immunodeficiency virus. Chest radiography performed in 54 patients was unremarkable. No specific abnormalities were detected in full blood count examination. 
Median white blood cell count was $9.3 \times 10^{9}$ cells/L, with normal haemoglobin (median $12.5 \mathrm{~g} / \mathrm{dL}$ ) and platelets (median $345 \times 10^{9} / \mathrm{L}$ ) levels in all patients. Median erythrocyte sedimentation rate was found to be 10 (IQR 5-25) mm/50 mins in 25 patients. Median C-reactive protein (CRP) level was at $7.8 \mathrm{mg} / \mathrm{L}(\mathrm{IQR} 4-11 ; \mathrm{n}=12)$. Both median erythrocyte sedimentation rate and median CRP level were not exceptionally remarkable.

TST was performed in 51 patients, with a median size of positive TST of $10 \mathrm{~mm}$ (IQR 5-13). In these 51 patients, $51 \%$ had a TST size of $>10 \mathrm{~mm}$, though $94.1 \%$ were $<20 \mathrm{~mm}$ in induration (Fig. 2). AFB smears were positive in 2 of 44 patients (4.5\%), in which one patient had a positive TB culture (the other had no growth on culture). Mycobacterial culture positivity was noted in only 10 (19.2\%) out of 52 cases where mycobacterial cultures were performed, with Mycobacterium tuberculosis identified in 2 cases, Mycobacterium bovis in 1 case (probably related to Bacille Calmette-Guérin [BCG] vaccination), while NTM was identified in 7 cases (4 cases of Mycobacterium haemophilum, 2 cases of Mycobacterium fortuitum, and 1 case classified as NTM without further characterisation). Out of the 60 patients in our study, coagulase-negative Staphylococcus deemed to be contaminants grew on bacterial cultures from 2 (3.3\%) patients, while none of the patients had positive fungal cultures.

Histological examination of affected lymph nodes in our 60 patients revealed the presence of necrosis in $56(93.3 \%)$. Positive AFB stains were found in $10(18.9 \%)$ patients $(n=53$; 1 with TB confirmed on culture, and 2 with NTM confirmed on culture), and fungal stains were negative for all lymph nodes studied. Tuberculosis polymerase chain reaction (PCR) was positive in $11(32.4 \%)$ out of 34 patients. In these 11 patients with positive PCR, 1 had a positive mycobacterial culture for TB, 1 had a positive culture for BCG, and 2 had positive cultures for NTM; the rest had no positive cultures.

In the 15 patients where TB was either confirmed $(n=2)$ or presumed ( $n=13$ ), complete excision biopsy, followed by antituberculous therapy, was administered and no recurrences were reported. The diagnosis of TB was based on positive cultures $(n=2)$, positive AFB smear on microbiological examination $(n=2)$, positive TB PCR on histological examination $(n=5)$, the presence of caseous necrosis on histological examination $(n=11)$, positive AFB stain on histological examination $(n=3)$, remote contact with household members with TB ( $\mathrm{n}=4$ cases), and/or TST size $>15 \mathrm{~mm}$ ( $\mathrm{n}=4$, of which one had vesiculation). The anti-TB treatment administered in our study consisted of two months of isoniazid, rifampicin and pyrazinamide (with one patient receiving quadruple initial phase therapy with the addition of ethambutol) followed by four months of isoniazid and rifampicin.

In the seven patients with confirmed NTM, complete and incomplete excision biopsies were done in three and two patients, respectively, while incision and drainage was done in three patients. These patients also received various combinations of clarithromycin/doxycycline/rifampicin for 6-12 weeks. Recurrence was noted in the two patients who underwent incomplete excision. These two patients subsequently underwent repeat complete excision with no further recurrences.

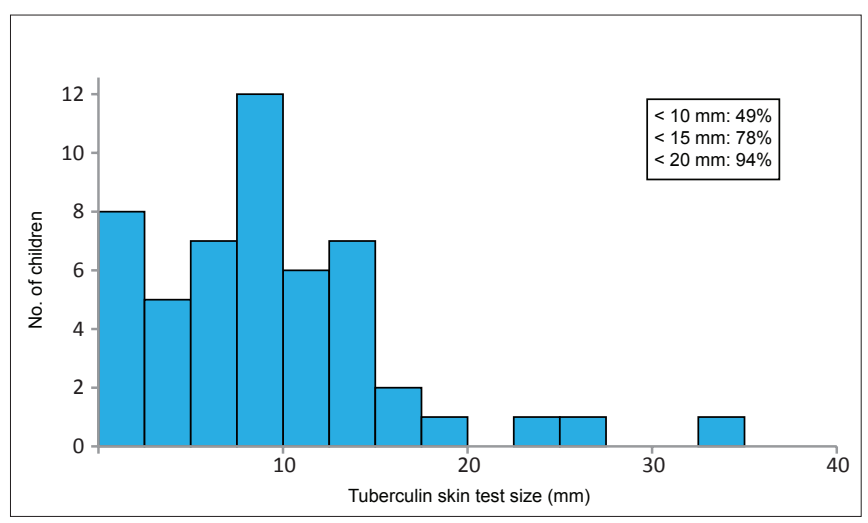

Fig. 2 Graph shows the size distribution of tuberculin skin tests performed in children with granulomatous cervicofacial lymphadenitis.

In our two patients with confirmed or presumptive BCG-associated lymphadenitis, one underwent complete excision at presentation and had no recurrences. The other patient underwent incision and drainage at presentation with subsequent wound breakdown, fistula formation and persistent purulent discharge. This second patient underwent repeat complete excision with no subsequent recurrences.

Of the remaining 36 patients with presumptive NTM infection, 23 underwent complete excision biopsy at presentation while 13 underwent either incision and drainage or incomplete excision. These patients also received various combinations of clarithromycin/ethambutol/rifampicin/isoniazid for a 2-12-month period. Recurrences were noted in three and six patients who underwent complete excision biopsy and incision and drainage/ incomplete excision, respectively. These recurrences were subsequently successfully managed by further complete excision or incision and drainage.

There were no particular clinical or laboratory characteristics in our study that reliably differentiated patients with TB and those with NTM confirmed on culture (Table I). Of particular interest was the fact that out of the three patients with cultureconfirmed NTM GCL who had TB PCR performed, 2 were positive (suggesting reduced specificity).

With regard to disease recurrence, Table II summarises the results of regression analysis for selected characteristics found to be significant or close to significant on univariate analysis. In the prediction of further recurrence, we found that initial complete excision proved to be the most successful intervention (odds ratio [OR] 0.11, 95\% confidence interval [Cl] 0.03-0.48). Age $<5$ years at presentation was found to be associated with a higher risk of recurrence (OR 14.14, 95\% Cl 1.69-118.42).

\section{DISCUSSION}

To the best of our knowledge, the present study is the first to describe the clinical and microbiological epidemiology of patients with GCL in Singapore, as well as the impact of treatment on patient outcomes. Although we noted an increasing frequency of GCL in recent years, we were unable to determine in the absence of appropriate total referral data whether a true increase in incidence had occurred, but we felt that this was likely due to increased awareness and referral. Also, while the microbiological 
Table I. Selected clinical and laboratory differences between culture-positive tuberculosis (TB) and nontuberculous mycobacteria (NTM) in children with granulomatous cervicofacial lymphadenitis.

\begin{tabular}{|c|c|c|c|}
\hline \multirow[t]{2}{*}{ Characteristic } & \multicolumn{2}{|c|}{ No. of patients (\%) } & \multirow[t]{2}{*}{ p-value } \\
\hline & TB $(n=2)$ & NTM $(n=7)$ & \\
\hline Age* (mths) $^{*}$ & $99(42-156)$ & $33(22-105)$ & 0.24 \\
\hline $\begin{array}{l}\text { Presence of multiple } \\
\text { affected lymph nodes }\end{array}$ & $0(0)$ & $5(71.4)$ & 0.22 \\
\hline $\begin{array}{l}\text { Parotid/submandibular } \\
\text { location of lymph nodes }\end{array}$ & $1(50)$ & $5(71.4)$ & 0.22 \\
\hline TST induration* $(\mathrm{mm})$ & $12^{+}$ & $14.5(11-21.7)$ & 0.48 \\
\hline $\mathrm{ESR}(\mathrm{mm} / 50 \mathrm{mins})$ & $54(3-105)$ & $25(8-38)$ & 1 \\
\hline $\begin{array}{l}\text { Presence of AFB on } \\
\text { histological staining }\end{array}$ & $1(50)$ & $2(33.3)$ & 0.64 \\
\hline TB PCR positive ${ }^{\S}$ & $1(100)$ & $2(66.6)$ & 0.75 \\
\hline $\begin{array}{l}\text { AFB smear positive on } \\
\text { microbiology }\end{array}$ & $1(100)$ & $0(0)$ & 0.14 \\
\hline
\end{tabular}

*Data is presented as median (interquartile range). tOnly one of two patients underwent TST, with vesicles formed. \#Testing was performed in only six patients in the NTM group. §Testing was performed in only one and three patients in the TB and NTM groups, respectively. ITesting was performed in only one and six patients in the TB and NTM groups, respectively.

AFB: acid-fast bacilli; PCR: polymerase chain reaction; TST: tuberculin skin test

Table II. Selected clinical and treatment characteristics associated with lymphadenitis disease recurrence.

\begin{tabular}{|c|c|c|c|c|}
\hline \multirow[t]{2}{*}{ Characteristic } & \multicolumn{2}{|c|}{ No. of patients (\%) } & \multicolumn{2}{|c|}{ p-value } \\
\hline & $\begin{array}{c}\text { With } \\
\text { recurrence } \\
(n=12)\end{array}$ & $\begin{array}{l}\text { Without } \\
\text { recurrence } \\
(n=48)\end{array}$ & Univariate & Multivariate \\
\hline Age $<5$ yrs & $11(91.7)$ & $21(43.8)$ & 0.003 & 0.008 \\
\hline $\begin{array}{l}\text { Multiple lymph } \\
\text { nodes affected }\end{array}$ & $6(50.0)$ & $6(12.5)$ & 0.04 & NS \\
\hline Tenderness & $6(50.0)$ & $13(27.1)$ & 0.15 & NS \\
\hline Warmth & $3(37.5)$ & $5(10.4)$ & 0.18 & NS \\
\hline $\begin{array}{l}\text { Initial complete } \\
\text { excision }\end{array}$ & $3(37.5)$ & $36(75.0)$ & 0.002 & 0.003 \\
\hline
\end{tabular}

NS: not significant on logistic regression analysis

yield in our study was low compared to the $50 \%-60 \%$ yield described in the literature, we established that our isolates were mainly derived from Mycobacterium haemophilum or Mycobacterium fortuitum, unlike in developed countries where Mycobacterium avium-intracellulare complex predominate..$^{91}$ Although the reasons for the low yield in our present study were not immediately obvious, it could have been related to sampling issues.

It is worth noting that there was an absence of any positive mycobacterial cultures for Mycobacterium avium-intracellulare complex in our cohort, and we were unable to explain why the microbial epidemiology in our cohort was different from that in North American or European cohorts. ${ }^{(9)}$ NTM are ubiquitous in the environment, existing in soil and water (including tap water), and ingestion of contaminated material has been thought to be the principal route of cervicofacial infection in children. ${ }^{(9,11,13)}$ Possible reasons for the microbiological differences noted could be due to: (a) true geographic differences with regard to mycobacterium epidemiology; (b) differing virulence factors causing a similar clinical disease; or (c) differing host susceptibility factors. These possible reasons require further study.

We were unable to demonstrate significant differences in clinical or laboratory characteristics between patients with positive TB culture and those with positive NTM culture, but this is likely due to the small sample size of positive cultures in our study. However, we noted that patients with NTM GCL in our study were commonly $<5$ years and more likely to have multiple lymph nodes in the preauricular/parotid or submandibular region, which was similar to that seen in previous reports. ${ }^{(7,10)}$ Many of the patients in our study did not have any systemic symptoms of TB, a positive TB contact history, or abnormal chest radiography. As noted in the earlier reports, TST values may not be helpful TST size $>15 \mathrm{~mm}$ was noted in some patients with confirmed NTM in our study, indicating the presence of cross-reactivity. Also, TB PCR can exhibit a false positive, as seen in two of our patients with positive TB PCR and a positive culture for NTM. It is important to recognise the two aforementioned points, so as to avoid unnecessary or inappropriate treatment or contact tracing for children with positive TST. Although cross-reactivity can occur, it is hoped that the advent and increased use of interferon-gamma release assays in children may address the issues with diagnostic specificity, since the assays are less likely to exhibit false positives. ${ }^{(18)}$

In the present study, we found that age $<5$ years at presentation was associated with a higher disease recurrence rate, while complete excision biopsy was associated with the lowest recurrence rate in patients with GCL. Four large, retrospective studies have demonstrated that surgical excision of infected nodes has a cure rate between $81 \%$ and $92 \%$, which rises to $95 \%$ if there is early surgical intervention. ${ }^{(19-22)} \mathrm{A}$ recent, well-conducted trial in which 100 children were randomised to either undergo surgical excision or receive at least 12 weeks of therapy with clarithromycin and rifabutin has shown surgery to be more effective than antibiotic therapy, with cure rates of $96 \%$ and $66 \%$, respectively. ${ }^{(23)}$ However, there was a reported complication rate of $28 \%$ in children who underwent surgery, which included secondary staphylococcal infection and transient or permanent facial nerve damage.

Perhaps because of the complications associated with surgical treatment, other authors have espoused a more conservative approach. Zeharia et al published their observations of 92 confirmed NTM cases over a 14-year period and concluded that, based on the natural history of disease resolution in their study, an observational approach can be effective in immunocompetent children. ${ }^{(24)}$ Closer review of Zeharia et al's paper shows that there was no response to single or multiple antibiotic therapies in almost all the children studied, and a majority of their patients had suppuration with purulent discharge for 3-8 weeks. ${ }^{(24)}$ Zeharia et al found that total resolution of symptoms was noted within six months in only $71 \%$ of patients, with full recovery in the remaining patients within one year, and their justification for this approach is 
patient/parental acceptability of the natural history of the disease and the presence of evidence of complete resolution on longterm follow-up. ${ }^{(24)}$ In an accompanying commentary, Starke remarked that the "question remains whether most parents and physicians will tolerate 6-12 months of an unsightly draining lesion in the face or neck, and whether the cosmetic outcome is as good as or better than when a skilled surgeon performs an early excision". ${ }^{25)}$ Based on the findings from our present study, we concur that complete excision at presentation would be preferred over conservative management or even incision and drainage, although we acknowledge that more randomised control studies on this would be required.

There were several limitations in our study. First, our study had a small sample size, together with the small number of patients with positive culture, which limited meaningful comparison between patients with TB and those with NTM. Second, possible selection bias could have occurred, as patients with a positive culture or in whom the disease behaved clinically like TB or NTM GCL might not have had a histological examination. Third, we did not have a record of the trend of the total number of lymphadenitis cases referred to our hospital during the study period. Thus, we were unable to determine whether there was a real increase in the incidence or rate of $\mathrm{GCL}$ in recent years. Fourth, the retrospective nature of our study limits the reliability of our conclusions regarding treatment efficacy. Fifth, varying follow-up periods could have led to uncertainty in actual recurrence rates. Although we searched hospital records for all subsequent in- and outpatient visits to determine whether recurrence could have occurred, there is the possibility that some patients could have experienced recurrence but sought medical opinion or treatment in another medical facility. Further local prospective studies are needed to address some of the above issues.

In conclusion, we found that children presenting with NTM GCL were more likely to be of a younger age, with more than one affected lymph node in the parotid or submandibular region. In prognosticating outcome, age below five years was predictive of recurrence, and complete excision of the affected node at initial presentation predicted non-recurrence. While recommendations regarding diagnosis and treatment exist, most were derived from small Caucasian populations. Further studies, using standardised inclusion criteria, to stratify patients in different risk groups into different treatment arms are needed.

\section{ACKNOWLEDGEMENTS}

We acknowledge the support of Duke-NUS/SingHealth Academic Medicine Research Institute and Taara Madhavan, Associate, Duke-NUS Graduate Medical School, Singapore, for her assistance in editing this manuscript.

\section{REFERENCES}

1. Gosche JR, Vick L. Acute, subacute, and chronic cervical lymphadenitis in children. Semin Pediatr Surg 2006; 15:99-106.

2. Chesney PJ. Cervical Lymphadenitis and Neck Infections. In: Long SS, Pickering LK, Prober CG, eds. Principles and Practice of Pediatric Infectious Diseases, 2nd ed. Philadelphia, PA: Churchill Livingstone, 2003: 165-76.

3. Moussatos $\mathrm{GH}$, Baffles TG. Cervical masses in infants and children. Pediatrics 1963; 32:251-6.

4. Lake AM, Oski FA. Peripheral lymphadenopathy in childhood. Ten-year experience with excisional biopsy. Am J Dis Child 1978; 132:357-9.

5. Benjamin DR. Granulomatous lymphadenitis in children. Arch Pathol Lab Med 1987; 111:750-3.

6. Knight PJ, Mulne AF, Vassy LE. When is lymph node biopsy indicated in children with enlarged peripheral nodes? Pediatrics 1982; 69:391-6.

7. Lai KK, Stottmeier KD, Sherman IH, McCabe WR. Mycobacterial cervical lymphadenopathy. Relation of etiologic agents to age. JAMA 1984; 251:1286-8.

8. Robson CD. Imaging of granulomatous lesions of the neck in children. Radiol Clin North Am 2000; 38:969-77.

9. Albright JT, Pransky SM. Nontuberculous mycobacterial infections of the head and neck. Pediatr Clin North Am 2003; 50:503-14.

10. Wolinsky E. Mycobacterial lymphadenitis in children: a prospective study of 105 children nontuberculous cases with long-term follow-up. Clin Infect Dis 1995; 20:954-63.

11. Wallace RJ Jr, Cook JL, Glassroth J, et al. Diagnosis and treatment of disease caused by nontuberculous mycobacteria. This official statement of the American Thoracic Society was approved by the Board of Directors, March 1997. Medical Section of the American Lung Association. Am J Respir and Crit Care Med 1997; 156:S1-S25.

12. Henriques B, Hoffner SE, Petrini B, et al. Infection with Mycobacterium malmoense in Sweden: a report of 221 cases. Clin Infect Dis 1994; 18:596-600

13. Thavagnanam S, McLoughlin LM, Hill C, Jackson PT. Atypical mycobacterial infections in children: the case for early diagnosis. Ulster Med J 2006; 75:192-4.

14. Grange JM, Yates MD, Pozniak A. Bacteriologically confirmed nontuberculous mycobacterial lymphadenitis in south east England: a recent increase in the number of cases. Arch Dis Child 1995; 72:516-7.

15. Jindal N, Devi B, Aggarwal A. Mycobacterial cervical lymphadenitis in childhood. Indian J Med Sci 2003; 57:1-15.

16. Huebner RE, Schein MF, Cauthen GM, Geiter LJ, O'Brien RJ. Usefulness of skin testing with mycobacterial antigens in children with cervical lymphadenopathy. Pediatr Infect Dis J 1992; 11:450-6.

17. Starke JR. Management of nontuberculous mycobacterial cervical adenitis. Pediatric Infect Dis J 2000; 7:674-5.

18. Starke JR. Interferon-gamma release assays for diagnosis of tuberculosis infection in children. Pediatr Infect Dis J 2006; 25:941-2.

19. Hazra R, Robson CD, Perez-Atayde AR, Husson RN. Lymphadenitis due to nontuberculous mycobacteria in children: presentation and response to therapy. Clin Infect Dis 1999; 28:123-9.

20. White MP, Bangash H, Goel KM, Jenkins PA. Non-tuberculous mycobacterial lymphadenitis. Arch Dis Child 1986; 61:368-71.

21. Fergusson JA, Simpson E. Surgical treatment of atypical mycobacterial cervicofacial adenitis in children. Aust NZ J Surg 1999; 69:426-9.

22. Makhani S, Postlethwaite KR, Renny NM, Kerawala CJ, Carton AT. Atypical cervico-facial mycobacterial infections in childhood. Br J Oral Max Surg 1998; 36:119-22.

23. Lindeboom JA, Kuijper EJ, Bruijnesteijn van Coppenraet ES, Lindeboom R, Prins JM. Surgical excision versus antibiotic treatment for nontuberculous mycobacterial cervicofacial lymphadenitis in children: a multicenter, randomized controlled trial. Clin Infect Dis 2007; 44:1057-64.

24. Zeharia A, Eidlitz-Markus T, Haimi-Cohen Y, et al. Management of nontuberculous mycobacteria-induced cervical lymphadenitis with observation alone. Pediatr Infect Dis J 2008; 27:920-2.

25. Starke JR. Commentary: The natural history of nontuberculous mycobacterial cervical adenitis. Pediatr Infect Dis J 2008; 27:923-4. 